\title{
CAUSES OF "BLACKOUTS" IN A MILITARY SETTING
}

\author{
Lieutenant-Colonel J. D. CORMACK, F.R.C.P.E., R.A.M.C. \\ British Military Hospital, Singapore
}

\section{Introduction}

THE assessment and investigation of transient loss of consciousness is a problem which faces all general practitioners and many physicians. The academic possibilities underlying such presentations are fully discussed in standard text books of medicine and Table I details one group of causes taken from Davidson (1966).

However the pattern of referral of such patients to hospital will vary in different types of practice. This will be determined by the patients' individual response to the occurrence and the presence of special environmental hazards, which will influence both the observers', and the doctors' view of the potential significance of the event. It was considered that these special problems of selection must influence the experience of this problem in Army Medicine. In the Army the number of personnel trained in bearing weapons and the large number involved in communications and in driving give particular significance to the occurrence of episodes of loss of consciousness.

It was decided to investigate all the adult admissions presenting with " blackouts" to the British Military Hospital (B.M.H.), Rinteln, in Germany for one year in a prospective study, and, to review the case notes of the admissions for "blackouts" in the previous year. The aims of the investigations were to assess the frequency with which organic disease, and, epilepsy were responsible for this type of presentation. A secondary aim was to look at the various facets of the cases to try to assess whether any group characteristics existed, and, to determine whether any such characteristics might be of assistance in diagnosis.

\section{Pattern of investigation}

The pattern of investigation used for the prospective study is detailed in Table II. This was also used to form the basis for abstracting information from the records of the previous year.

Number of cases. Nineteen patients were admitted to the prospective study. Twentythree were available for assessment from the preceding year.

Age distribution. The age distribution of the total studied, that is forty-two patients is detailed in Table III. This appears to reflect the age distribution within the population at risk.

Sex distribution. Thirty-six men and six females were included in the two year period. The number of soldiers approximately equals the total number of dependants in the population which is being served by the hospital. When the number of children is allowed for; the implication is that there is likely to be an excess of males. However this is not in the proportion of $36: 6$. Male patients must either be markedly more likely to develop transient loss of consciousness or are referred for admission on a different set of criteria. Both of these factors are possibly true, but, the latter is considered the more important in this study. 
Table I

Range of causes of loss of consciousness

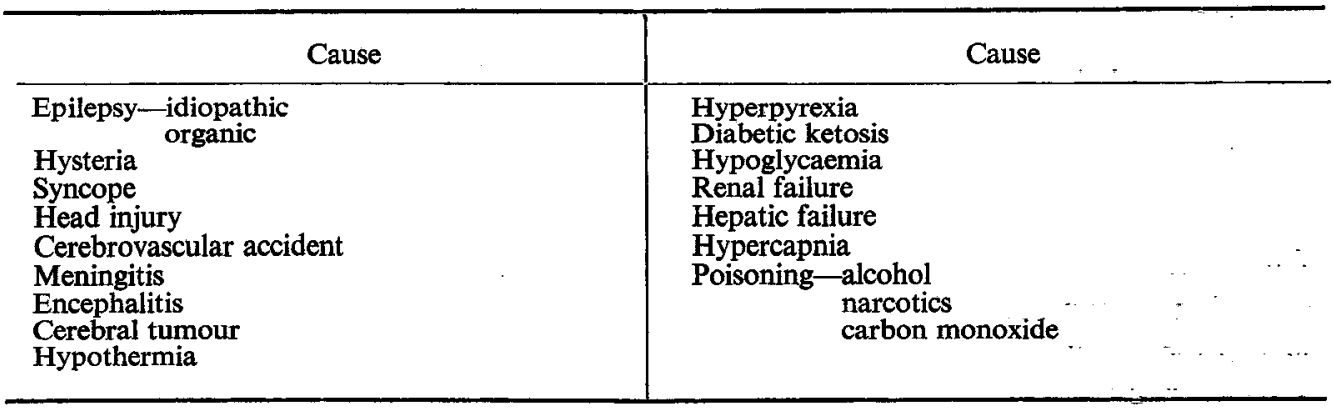

Table II

Pattern of investigation

Family

Previous health

History

Present health

Present accident

Findings on clinical examination

age, sex, marital status, employment, personality

at time of accident

between occurrence

Special investigations

Haematology (E.S.R., Hb, W.B.C. \& Diff:)

Blood urea, electrolytes, S.G.O.T., S.G.P.T.

Urinalysis, microscopy, culture of urine

Radiology of chest, skull

V.D.R.L.

c.s.f. examination

Fasting and random blood sugar

e.e.g. tracing

Table III

Age distribution

\begin{tabular}{c|c|c|c}
\hline Age group in years & Epilepsy & Other diagnosis & Totals \\
\hline 15 to 20 & 6 & 8 & 14 \\
20 to 25 & 9 & 11 & 20 \\
25 to 30 & 2 & 2 & 4 \\
30 to 35 & 2 & 1 & 3 \\
45 to 50 & - & 1 & 1 \\
\hline
\end{tabular}

Marital status. Thirteen of the forty-two patients were married; and twenty-nine were single. Only fourteen of the patients were under twenty years of age and twenty-eight were over twenty. Less than half of those over twenty in this group of patients were married. This rather small proportion of married patients most probably reflects the rather limited scope for contact with English speaking girls for many soldiers serving in Germany.

Employment. The range of employment of these patients is detailed in Table IV: The largest group, twelve out of the forty-two patients, were drivers, and the second largest group were infantry, or, general duty personnel. There were eight in this section. The remainder were more widely scattered representing a range of different jobs.

The group, which is over-represented, is the driver section. This suggests that transient loss or impairment of consciousness is taken seriously by both the individual 
Table IV

Employment

\begin{tabular}{l|c|c|c}
\multicolumn{1}{c|}{ Employment } & Epilepsy & Other diagnosis & Totals \\
\hline Driver & 6 & 6 & 12 \\
Infantry/general duties & 4 & 4 & 8 \\
Clerk & 2 & 2 & 4 \\
Technician & 1 & 4 & 3 \\
Cook & 2 & 2 & 2 \\
Housewife & 2 & 1 & 3 \\
Teacher/nurse & 1 & 3 & 3 \\
Storeman & 1 & 1 & 2 \\
\hline
\end{tabular}

drivers, who report sick, and also by their referring doctors. This bias, in favour of drivers being referred for investigation, represents a form of selection according to special responsibility, where ill health could involve risks to many people. It is probable that similar incidents in other jobs are not always referred for investigation.

Length of history. Many of the patients gave a history of fairly numerous episodes over very variable periods of time. Seven patients were admitted, with a history of less than one week in duration. Three patients had a history of more than one week and less than one month in duration. Fourteen cases had a history of several months duration (all less than one year). Sixteen cases had histories of longer than one year. In two cases assessed retrospectively the length of history had not been recorded in the notes.

The length of the history overlaps with the number of previous episodes, which is considered below. It was surprising to find so many patients in such a small series with such complicated histories: both with regard to length and number of previous episodes.

Number of episodes. The number of episodes of loss of consciousness varied remarkably. This is summarised in Table $\mathrm{V}$.

Table $\mathrm{V}$

Number of previous episodes

\begin{tabular}{c|c|c|c}
\hline Number & Epilepsy & Other diagnosis & Totals \\
\hline 1 & 4 & 9 & 13 \\
2 & 1 & 4 & 5 \\
3 & 1 & 4 & 5 \\
4 & 1 & 1 & 2 \\
5 & -8 & 1 & 9 \\
Several & 4 & - & 4 \\
Very many & 4 & \\
\hline
\end{tabular}

The case records of two patients, from the preceding year contained no information about the number of blackouts. Virtually a third of the group were referred after a single episode. A similar proportion were referred after many episodes. The remainder were investigated after two to six episodes of loss of consciousness.

Clinical assessment of the "blackout". The clinical features led to the "blackout" being described as showing epileptic features in thirteen instances. Six patients clearly had simple vaso-vagal faints. Five patients were hysterical and underlying manipulative 
endeavour was uncovered. The remainder were grouped as indeterminate, on the basis of the clinical history and in the absence of adequate accounts by witnesses.

The features recorded in relation to epileptic attacks were a well defined aura or prodromal experience ( 5 cases) a cri epilepticus ( 1 case) tongue biting ( 8 cases) or in urinary incontinence ( 9 cases); well defined tonoclonic phases ( 7 cases) or a Jacksonian attack ( 1 case). The finding of a Babinski response in the recovery phase was looked for, and, a history of severe headache, muscular fatigue and undue somnolence, in the recovery phase, was noted (8 cases).

A background of alcoholic excess (5 cases), excessive fatigue, or emotional tension was looked for ( 5 cases). A family history of epilepsy was given by two patients.

The relationship between a "blackout" showing epileptic feature, where only a single attack has occurred, and a diagnosis of epilepsy requires further comment. It is clear that the setting of the attack is important. The patient, who feels frightened and unwell in the dentist chair, and who faints during the local anaesthetic injection may well show clonic movements and urinary incontinence, following cerebral hypoxia if the dentist proceeds to extraction with the patient still semi-upright in the chair. To suggest therefore that epileptic features imply epilepsy is wrong.

The diagnosis of epilepsy was not made unless more than one "blackout" had occurred, irrespective of its nature. This is commented upon further in relation to disposal of the cases.

Personality assessment. In addition to the impression each patient made while in hospital, his previous work record was investigated and his regimental officer's opinion was recorded. Adequate information was obtained for the nineteen patients in the prospective group and for fifteen patients from the preceding year. These fifteen were still attending medical out patients for follow-up or for boarding.

Fourteen were considered to have "good" personalities. These were people, who were characterised by all or most of the following features. They tended to have good work records before joining the Army. They were free from histories of previous neurotic behaviour. They had stable marriages. Their military record showed consistency of application. They were valued by their regimental officers. In hospital they generally showed acceptance of the situation; while retaining a need for reasonable explanation of what was happening and a drive towards discharge from hospital in a normal category (if they desired to remain in the Services). Thirteen were considered to be only "fair" and the remainder were called "poor". They were seven in number. The " poor" personality group showed a very different group of characteristics. They frequently had had a variety of different jobs before joining the Army. They had generally not held any one job for any length of time. There was frequently a history of both neurotic illness with slow recovery and minor disciplinary offences. Their social habits tended to be irregular with regard to alcohol intake. They tended to have, if married, an undue amount of domestic strife. They were often considered unreliable by their regimental officers. In hospital they tended to either show off, or, be querulous and unreasonable, frequently requiring repeated explanation, to which they tended not to listen. They frequently showed manipulative drive: trying to escape from courses, onerous duties, or exercises; to obtain a new house, a new posting or a discharge from the Army. The people with "fair" personalities showed some features of both groups and could not reasonably be placed in either of the previous categories. 
When these thirty-four cases are divided according to final diagnosis a distinction can be seen. The cases diagnosed as epilepsy are separated from other diagnosis in Table VI and listed according to personality. In this series, at the time of diagnosis, the group

Table VI

Personality assessment of patients

\begin{tabular}{c|c|c}
\hline Personality & Epilepsy & Other diagnosis \\
\hline Good & 10 & 4 \\
Fair & 5 & 8 \\
Poor & 2 & 5 \\
\hline
\end{tabular}

with epilepsy were predominantly considered to be of "good" personality. The other causes of " blackouts" were more likely to be associated with a "poor" or "fair" type of personality.

\section{Findings on investigation}

The features relating to the character of the "blackout" have already been discussed. They were elicited from the history: given either by the patient or by witnesses. Only very occasionally does the opportunity of seeing an attack arise.

F. Med.138s were generally available. They were not considered as valuable as a statement obtained in personal interview of a witness. The relatively poor value of the F. Med. 138 is attributed to its apparent complexity. The lay witness, most frequently a soldier or a wife, is faced with numerous questions about the incident and feels that he doesn't remember or cannot describe the details asked. His spontaneity is lost. He obtains help, frequently (in my opinion) from the orderly or medical assistant. This appears to result in the use of semi-medical jargon in the report. However, this use of language may in fact distort the spontaneous description. The value of the $F$. Med. 138 is lessened when it is completed in retrospect.

It would perhaps be most useful if the F. Med. 138 were taken as a reminder of the need to obtain a careful description of what happened. The medical officer should interview the witness and record the event as far as possible in the language used by the witness. The weakness of a form lies in the ease with which it can be handed out, and responsibility delegated, with the unhelpful instructions "fill that in".

Clinical examination in this group was not helpful. This was carried out between episodes. The medical officers referring the cases rarely recorded the state of the pupils or the plantar response when the patient had been seen after an attack. Routine haematology, (blood film, haemoglobin, erythrocyte sedimentation rate) urinalysis, V.D.R.L., X-rays of chest and skull in all cases, c.s.f. examination and lumbar puncture findings in the thirty-one cases so examined, and biochemistry including blood sugar (fasting and random values) electrolytes, blood urea and transaminase estimations, again in all cases, were all within the normal range.

This implies that no case of organic or secondary epilepsy was uncovered during the investigation of this series. However, the list of negative findings does not mean that investigation of such cases is unnecessary. A high level of suspicion requires to be main- 
tained as cases of secondary disease, or generalised diseases will occur, presenting with loss of consciousness, and will be missed unless careful investigation is carried out when indicated.

Electro-encephalogram tracings had been obtained in the majority of the patients managed at B.M.H. Rinteln, and had been reported by a neurologist. In twenty instances they were considered essentially physiological. In six cases they were assessed as unstable, and in thirteen records, patterns consistent with epilepsy were described.

\section{Diagnosis}

In the absence of any organic state found in this group, the diagnosis reached fell into three main categories.

The first is of idiopathic epilepsy. This diagnosis was reached in nineteen patients in the group, that is forty-five per cent. When the two years of the survey are separated the diagnosis was made in ten cases out of the twenty-three from the previous year and in nine cases out of the nineteen studied in the prospective year. Epilepsy was diagnosed with almost similar frequency in the two years of the study. All the cases of epilepsy had had multiple episodes of unconsciousness, associated with epileptiform features. Thirteen of them had e.e.g. tracings with epileptic pattern and two with unstable pattern. The remaining e.e.g. tracings were normal.

Of the remaining cases, eight were considered to be related to personality factors: grouped together as immaturity reactions or hysterical behaviour patterns. In two cases the underlying process was considered to be syncope. Both of these groups were frequently associated with some environmental stress. This was varied and might range from emotional situations at work or at home, the presence of a hangover, excessive fatigue, hunger, or, exposure to unpleasant or stressful conditions such as may occur on exercises. Five cases did not fit into either of the above groups and were kept under close review. They are discussed further under disposal of the cases.

\section{Disposal of the cases}

Seventeen of the nineteen cases of epilepsy were boarded P8. Of the remaining cases, two (one teacher, one housewife) were treated locally and followed up at the out patient department.

Five cases were boarded P7(HO) fit for restricted duty, and were followed up closely at medical out patients. These consisted of two patients with only one episode, associated with epileptiform features, which were not explicable on environmental factors, and were also associated with an unstable e.e.g. pattern. It was felt that these cases were probably epileptic, but, that the diagnosis should not be made unless further episodes occurred.

The remaining three cases boarded $\mathrm{P} 7(\mathrm{HO})$ were individuals witn numerous occurrences. These were of indeterminate nature. The individuals appeared reasonably stable and there was no clear environmental stress or manipulative aim in their behaviour. It was hoped to obtain better witnessed accounts of their blackouts; to follow their e.e.g. tracing and perhaps to uncover underlying emotional disturbances.

Eighteen cases were returned to duty in a normal category, fit for full duties. 


\section{The features of the epileptic group}

Epilepsy occurred in forty-five per cent of the cases in this series and must therefore be considered in all cases of repeated transient loss of consciousness.

The distribution of the epileptic patients was compared with the distribution of the other cases in respect of age, sex distribution, marital status, type of employment. No significant difference for any of these factors was demonstrated. The age and employment comparison is detailed in Tables III and IV. There were seventeen men and two females in the epileptic group and nineteen men and four females in the remaining group. Six of the epileptic patients were married (out of nineteen) and seven of the remaining cases (out of twenty-three) were married.

It has already been demonstrated that the epileptic patients personality assessment was, in general, significantly better than the personality assessment of the other cases. The second point of difference relates to the number of previous episodes. The epileptic group, tended to be characterised by a history of a larger number of previous episodes than the cases with other diagnosis. This is detailed in Table V. This must be regarded as a relative distinction, as any one case of epilepsy may be diagnosed in their second, third or any one subsequent attack. However, within the range of cases seen in any series this distinction is likely to be valid on several occasions.

\section{Conclusions}

Within the limitations of this study, in which a relatively small number of cases have been investigated over a two year peiod, it is possible to draw some conclusions, which may well apply to the practice of medicine in other military populations and in relation to other military or service hospitals.

The incidence of organic disease states in patients from a military population presenting with blackouts is small. However, such cases will occur and this implies the need for a careful diagnostic approach and planned investigation whenever indicated.

Idiopathic epilepsy is responsible for a significant proportion of these cases. In addition to the classical clinical features of the epileptic attack and e.e.g. findings the diagnosis should be suspected when there is a history of fairly numerous episodes. This is true even when the individual episode of loss of consciousness, lacks any distinctive feature. When frequent attacks are occurring in an individual of good personality the possible diagnosis of epilepsy requires particularly careful consideration.

REFERENCE

Davidson, Sir S. (1966). The Principles and Practice of Medicine. 8th ed. E. \& S. Livingstone, Edinburgh. 